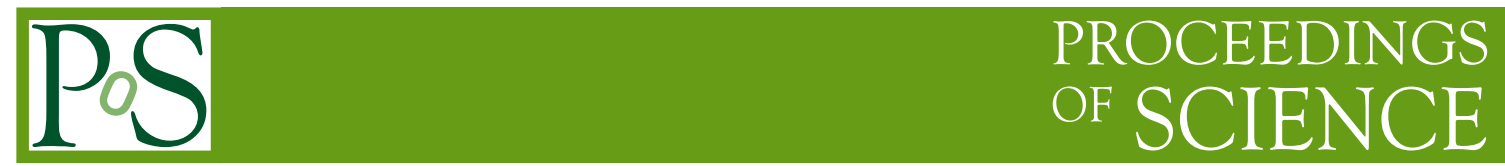

\title{
Study of strange matter production in the heavy ion collisions at NUCLOTRON
}

Vladimir Ladygin for the BM@N collaboration*

Joint Institute for Nuclear Research, Dubna, Russia

E-mail: vladyginejinr.ry

T.O.Ablyazimov ${ }^{1}$, J.Aichelin ${ }^{8}$, V.V.Andreev ${ }^{6}$, V.V.Avdejchikov ${ }^{1}$, A.V.Averianov ${ }^{1}$, S.V.Avramenko ${ }^{1}$, V.P.Akishina ${ }^{1}$, P.G.Akishin ${ }^{1}$, V.D.Aksinenko ${ }^{1}$, A.V.Alfeev ${ }^{1}$, V.S.Alfeev ${ }^{1}$, V.A.Babkin ${ }^{1}$, S.A.Baginyan ${ }^{1}$, V.P.Balandin ${ }^{1}$, A.V.Baranova ${ }^{11}$, M.I.Barznat ${ }^{9}$, S.N.Basylev ${ }^{1}$, D.V.Belyakov ${ }^{1}$, G.A.Bogdanovaa ${ }^{11}$, I.V.Boguslavsky ${ }^{1}$, I.A.Bolshakova ${ }^{22}$, E.E.Boos ${ }^{11}$, E.L.Bratkovskaya ${ }^{4}$, W.Cassing ${ }^{5}$, S.P.Chernenko ${ }^{1}$, V.Davkov $^{1}$, K.Davkov ${ }^{1}$, V.I.Demidova ${ }^{1}$, Z.Deng ${ }^{21}$, O.Yu.Derenovskaya ${ }^{1}$, S.V.Donskov ${ }^{13}$, V.B.Dunin ${ }^{1}$, A.A.Ershov ${ }^{11}$, O.V.Fateev ${ }^{1}$, Yu.I.Fedorov ${ }^{1}$, V.A.Gapienko ${ }^{13}$, O.P.Gavrishuk ${ }^{1}$, S.G.Genchev ${ }^{7}$, V.M.Golovatyuk ${ }^{1}$, A.I.Golokhvastov ${ }^{1}$, S.N.Golovnya ${ }^{13}$, N.Grigalashvili ${ }^{1}$, F.F.Guber ${ }^{12}$, K.K.Gudima ${ }^{9}$, S.Gumen ${ }^{22}$, Yu.V.Gurchin ${ }^{1}$, C.Hartnack ${ }^{8}$, V.Hayduchok ${ }^{22}$, J.Heuser ${ }^{2}$, X.Huang ${ }^{21}$, V.V.Ivanov ${ }^{1}$, A.P.Ivashkin ${ }^{12}$, A.P.lerusalimov' ${ }^{1}$, E.-M.llgenfritz ${ }^{1}$, A.Yu.Isupov ${ }^{1}$, V.Ivanets $^{22}$, M.Janek ${ }^{17}$, Yu.-T.Karachuk ${ }^{1,10}$, D.E.Karmanov ${ }^{11}$, V.D.Kekelidze ${ }^{1}$, G.D.Kekelidze ${ }^{1}$, V.S.Khabarov ${ }^{1}$, S.V.Khabarov ${ }^{1}$, A.G.Kholodenko ${ }^{13}$, A.N.Khrenov ${ }^{1}$, V.I.Kireev ${ }^{1}$, Yu.T.Kiryushin ${ }^{1}$, P.I.Kisel ${ }^{1}$, I.V. Kisel ${ }^{3}$, I.Kogut ${ }^{22}$, E.S.Kokoulina ${ }^{1,6}$, V.I.Kolesnikov ${ }^{1}$, V.Konchakovski ${ }^{5}$, B.Kopko ${ }^{22}$, M.G.Korolev ${ }^{11}$, A.M.Korotkova ${ }^{1}$, Y.Kost $^{22}$, N.Kovaljova ${ }^{22}$, V.E.Kovtun ${ }^{18}$, L.Kozachenko ${ }^{22}$, M.Kravets ${ }^{22}$, A.Kugler ${ }^{20}$, E.O.Kurbatov ${ }^{11}$, A.B.Kurepin ${ }^{12}$, A.K.Kurilkin ${ }^{1}$, P.K.Kurilkin ${ }^{1}$, V.Kushpil ${ }^{20}$, A.Yu.Kutov ${ }^{15}$, A.V.Kuznezov ${ }^{1}$, N.A.Kuzmin ${ }^{1}$, E.A.Ladygin ${ }^{1}$, V.P.Ladygin ${ }^{1}$, N.B.Ladygina ${ }^{1}$, A.A.Lebedev ${ }^{1,2}$, V.V.Lenok ${ }^{11}$, A.Levchenko ${ }^{22}$, Y.Li $^{21}{ }^{2}$ O.Linnyk ${ }^{5}$, D.Lipchinski $^{10}$, E.P.Litvinenko ${ }^{1}$, S.P.Lobastov ${ }^{1}$, I.P.Lokhtin ${ }^{11}$, J.Lukstins ${ }^{1}$, V.N.Lyashchenko ${ }^{19}$, D.T.Madigozhin ${ }^{1}$, O.Makido $^{22}$, N.V.Maksimenko ${ }^{6}$, A.I.Malakhov ${ }^{1}$, L.V.Malinina $^{11}$, M.Martinska ${ }^{16}$, M.M.Merkin ${ }^{11}$,Yu.V.Mikhaylov ${ }^{13}$, V.V.Mialkovsky ${ }^{1}$, A.Moroz $^{22}$, Yu.N.Murin ${ }^{1}$, S.N.Nagorny ${ }^{1}$, Y.Nakalov ${ }^{22}$, V.A.Nikitin ${ }^{1}$, P.V.Nomokonov ${ }^{1}$, 
O.V.Okhrimenko ${ }^{1}$, I.Palynyak ${ }^{22}$, V.V.Pavljuchkov ${ }^{1}$, A.N.Parfenov ${ }^{1}$, Yu.P.Petukhov ${ }^{1}$, V.N.Pechenov ${ }^{1}$, O.Yu.Pechenova ${ }^{1}$, V.D.Peshekhonov ${ }^{1}$, S.M.Piyadin ${ }^{1}$, V.A.Polyakov ${ }^{13}$, J.Popovichi ${ }^{10}$, I.S.Radkov ${ }^{7}$, S.V.Radnev ${ }^{7}$, L.Rakhlin ${ }^{22}$, A.M.Raportirenko ${ }^{1}$, G.Rashevski $^{7}$, S.N.Reva ${ }^{19}$, S.G.Reznikov ${ }^{1}$, V.N.Riadovikov ${ }^{13}$, P.A.Rukoyatkin ${ }^{1}$, I.A.Rufanov ${ }^{1}$, A.S.Sadovsky ${ }^{12}$, A.A.Semak ${ }^{13}$, P.Senger $^{2}$, A.V.Shabunov ${ }^{1}$, H.Shapovalov $^{22}$, V.Shejnast ${ }^{1}$, A.L.Shkilyov ${ }^{18}$, F.Shurygin ${ }^{22}$, A.V.Shutov ${ }^{1}$, V.M.Slepnev ${ }^{1}$, I.V.Slepnev ${ }^{1}$, A.M.Snigirev ${ }^{11}$, I.Sushko ${ }^{22}$, V.I.Suzdalev ${ }^{1}$, O.Svoboda $^{20}$, V.V.Syschenko ${ }^{14}$, S.Yu.Sychkov ${ }^{1}$, A.A.Terekhin ${ }^{1,14}$, A.V.Terlezky ${ }^{1}$, O.V.Teryaev ${ }^{1}$, V.V.Tiflov ${ }^{12}$, V.V.Tikhomirov ${ }^{1}$, P.Tlusty ${ }^{20}$, N.D.Topilin ${ }^{1}$, B.Trpisova ${ }^{17}$, Yu.P.Tsupa ${ }^{13}$, A.A.Turchin ${ }^{19}$, S.Tymoshyn ${ }^{22}$, M.N.Ukhanov ${ }^{13}$, E.A.Usenko ${ }^{1,12}$, J.Urban ${ }^{16}$, V.A.Vasendina $^{1}$, T.A.Vasiliev ${ }^{1}$, Yu.Vassiliev ${ }^{3}$, N.M.Vladimirova ${ }^{1}$, A.V.Vishnevsky ${ }^{1}$, I.E.Vnukov ${ }^{14}$, S.Vokal ${ }^{16}$, S.V.Volgin ${ }^{1}$, V.Yu.Volkov ${ }^{11}$, A.P.Vorobiev ${ }^{13}$, A.G.Voronin ${ }^{11}$, V.V.Voronyuk ${ }^{1}$, H.Voroshylo ${ }^{22}$, J.Wang ${ }^{21}$, Y.Wang ${ }^{21}$, V.Yerashok ${ }^{22}$, A.I.Yukaev ${ }^{1}$, Y.Zagachevskyi $^{22}$, I.I.Zaljyubovsky ${ }^{18}$, Yu.V.Zanevsky ${ }^{1}$, A.Zenkov ${ }^{7}$, X.Zhu ${ }^{21}$,

A.P.Zinchenko ${ }^{1}$, P.V.Zrelov ${ }^{1}$, V.N.Zryuev ${ }^{1}$ (BM@N collaboration)

${ }^{1}$ Joint Institute for Nuclear Research, Dubna, Russia

2 GSI Helmholtz-Zentrum fÃijr Schwerionenforschung, Darmstadt, Germany

${ }^{3}$ Goethe University, Frankfurt, Germany

${ }^{4}$ Institute for Theoretical Physics\& Frankfurt Institute for Advanced Studies, Goethe University,

Frankfurt, Germany

${ }^{5}$ Institute for Theoretical Physics, Gissen University, Germany

${ }^{6}$ Gomel State Technical University, Gomel, Belarus

${ }^{7}$ Laboratory for Technical Developments and Applications, BAS, Sofia, Bulgaria

${ }^{8}$ SUBATECH, Nantes University, Ecole des Mines, Nantes, France

${ }^{9}$ Institute of Applied Physics, ASM, Kishinev, Moldova

${ }^{10}$ Advanced Research Institute for Electrical Engineering, Bucharest, Romania

11 Skobeltsyn Institute of Nuclear Physics, Moscow State University, Moscow, Russia

${ }^{12}$ Institute for Nuclear Research of RAS, Moscow, Russia

${ }^{13}$ State Scientific Center of RF-Institute of High Energy Physics, Protvino, Russia

${ }^{14}$ Belgorod State University, Belgorod, Russia

${ }^{15}$ DM of Komi National Center, Ural Branch of RAS, Syktyvkar, Russia

${ }^{16}$ P.-J.Shafarik University, Koshice, Slovakia

${ }^{17}$ Physics Department, University of Zilina, Zilina, Slovak Republic

${ }^{18}$ V.N.Karazin Kharkov National University, Kharkov, Ukraine

${ }^{19}$ National Science Center Kharkov Institute of Physics and Technology, Kharkov, Ukraine

${ }^{20}$ Nuclear Physics Institute, Academy of Sciences of Czech Republic, Rez, Czech Republic

${ }^{21}$ Department of Engineering Physics, Tsinghua University, Beijing, China

${ }^{22}$ Lviv Polytechnic National University, Lviv, Ukraine 
It is proposed to install an experimental setup in the fixed-target hall of the Nuclotron with the final goal to perform a research program focused on the production of strange matter in heavyion collisions at beam energies between 2 and $6 \mathrm{~A} \mathrm{GeV}$. The basic setup will comprise a largeacceptance dipole magnet with inner tracking detector modules based on double-sided Silicon micro-strip sensors and GEMs. The outer tracking will be based on the drift chambers and straw tube detector. Particle identification will be based on the time-of-flight measurements. This setup will be sufficient perform a comprehensive study of strangeness production in heavy-ion collisions, including multi-strange hyperons, multi-strange hypernuclei, and exotic multi-strange heavy objects. These pioneering measurements would provide the first data on the production of these particles in heavy-ion collisions at Nuclotron beam energies, and would open an avenue to explore the third (strangeness) axis of the nuclear chart.

The extension of the experimental program is related with the study of in-medium effects for vector mesons decaying in hadronic modes. The studies of the $N N$ and $N A$ reactions for the reference is assumed.

XXI International Baldin Seminar on High Energy Physics Problems September 10-15, 2012

JINR, Dubna, Russia

${ }^{*}$ Speaker. 


\section{Strangeness at Nuclotron}

The Nuclotron at JINR will provide beams of heavy ions with energies up to $6 \mathrm{~A} \cdot \mathrm{GeV}$ for isospin symmetric nuclei, and $4.65 \mathrm{~A} \cdot \mathrm{GeV}$ for $A u$ nuclei. In central heavy-ion collisions at these energies, nuclear densities of about 4 times nuclear matter density can be reached. These conditions are well suited to investigate the equation-of-state (EOS) of dense nuclear matter which plays a central role for the dynamics of core collapse supernovae and for the stability of neutron stars. At the same time, heavy-ion collisions are a rich source of strangeness, and the coalescence of kaons with lambdas or of lambdas with nucleons will produce a vast variety of multi-strange hyperons or of light hypernuclei, respectively. Even the production of light double-hypernuclei or of doublestrange dibaryons is expected to be measurable in heavy-ion collisions at Nuclotron energies. The observation of those objects would represent a breakthrough in our understanding of strange matter, and would pave the road for the experimental exploration of the 3-rd dimension of the nuclear chart. These studies are complimentary to the CBM experimental program at SIS100 [i].

High-energy heavy-ion collisions offer the unique possibility to create and to investigate hot and dense nuclear matter in the laboratory. The nucleon densities in the collision zone of two gold

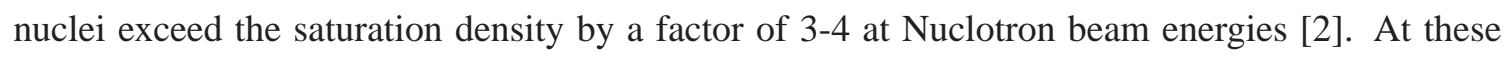
densities the nucleons start to overlap, and it was speculated that under such extreme conditions the onset of chiral symmetry restoration may occur while the quarks are still confined. In this case, the bulk properties (for example the energy density) are dominated by quarks which occupy the Fermi sea, whereas baryons represents the excited states. Due to the increase of the degrees-of-freedom with respect to hadronic matter this particular state can be regarded as a new phase of nuclear matter where quarks and baryons coexist. This so called "quarkyonic" phase [3] is predicted to be located at large baryo-chemical potentials and moderate temperatures, and, therefore, may be produced in heavy-ion collisions at Nuclotron beam energies.

The central question is which experimental observables are sensitive to the properties and the degrees-of-freedom of QCD matter at supra-nuclear densities. We suggest to study the yield and the phase-space distribution of multi-strange hyperons at beam energies close to their production threshold in nucleon-nucleon collisions. The threshold beam energies for reactions like $p p \rightarrow \Xi^{-} K^{ \pm} K^{ \pm} p$ or $p p \rightarrow \Omega^{-} K^{ \pm} K^{ \pm} K^{0} p$ are 3.7 or $7.0 \mathrm{GeV}$, respectively. However, $\Xi^{-}$and $\Omega^{-}$ hyperons can also be created via strangeness exchange reactions like $\Lambda \Lambda \rightarrow \Xi^{-} p$ and $\Lambda \Xi^{-} \rightarrow \Omega^{-} n$ or $\Lambda K^{-} \rightarrow \Xi^{-} \pi^{0}$ and $\Xi^{-} K^{-} \rightarrow \Omega^{-} \pi^{-}$. In these cases, the $\Lambda$ and the $K^{-}$have been produced in independent reactions such as $p p \rightarrow K^{+} \Lambda p$ and $p p \rightarrow K^{+} K^{-} p p$ which require only 1.6 and $2.5 \mathrm{GeV}$, respectively. Due to these multiple-step (or three body) collisions involving lambdas and kaons the production of multi-strange hyperons is expected to be enhanced at high densities, and their yield is sensitive to the baryon density reached in the fireball. Therefore, systematic measurements of $\Xi^{-}$ and $\Omega^{-}$hyperon production as function of beam energy and size of the colliding nuclei offer the possibility to study the nuclear matter equation-of-state, or baryon density fluctuations as they are expected to occur when the system undergoes a first-order phase transition. These fluctuations may also indicate the existence and the location of a QCD critical endpoint. Moreover, the energy distributions of multi-strange hyperons provide information on the fireball temperature and the radial flow at the time when they are emitted.

The proposed experiment would deliver the first data on the production of multi-strange hy- 
perons in heavy-ion collisions at Nuclotron beam energies. Also we propose to produce hypernuclei (single- and double-lambda) in heavy-ion collision via coalescence of lambdas with nucleons or light fragments. In central $A u+A u$ collisions at $4 \mathrm{~A} \mathrm{GeV}$ still 4 lambdas are produced. The probability for coalescence of lambdas with protons or light nuclei like $\mathrm{He}$, however, increases with decreasing beam energy, i.e. with decreasing temperature of the fireball, and, hence, increasing $\mathrm{He}$ yield. According to the estimations from ref. 凹, one can expect at $\mathrm{Nu}-$ clotron energies yield of $3 \cdot 10^{-8}{ }_{\Lambda \Lambda}^{6} \mathrm{He}, 10^{-6}{ }_{\Lambda \Lambda}^{5} \mathrm{H}$, and $2 \cdot 10^{-} 2{ }_{\Lambda}^{3} \mathrm{H}$ per central collision. Experimentally one has to reconstruct the decay chain of the hypernuclei (if it exists), for example: ${ }_{\Lambda \Lambda}^{5} H \rightarrow{ }_{\Lambda}^{5} \mathrm{He}+\pi^{-},{ }_{\Lambda}^{5} \mathrm{He} \rightarrow{ }^{4} \mathrm{He}+p+\pi^{-}$. The ${ }_{\Omega}^{6} H$ (if it exists) can be detected using the following chain ${ }_{\Omega}^{6} H \rightarrow{ }_{\square}^{6} H e+\pi^{-},{ }_{\square}^{6} H e \rightarrow{ }_{\Lambda \Lambda}^{6} H e \rightarrow{ }_{\Lambda}^{5} H e+p+\pi^{-},{ }_{\Lambda}^{5} H e \rightarrow{ }^{4} H e+p+\pi^{-}$. The systematic studies of neutron-rich and halo(loosely bound) hypernuclei $\left({ }_{\Lambda}^{3} \mathrm{H},{ }_{\Lambda}^{4} \mathrm{H},{ }_{\Lambda}^{6} \mathrm{He}\right.$ etc.) [阿 can be performed simultaneously. Part of these measurements requires the detection of light nuclear fragments ( for instance, ${ }^{4} \mathrm{He}$ ) what can be performed using amplitude information from STS (or from the scintillation detectors placed in front of $S T S$ ). The feasibility study will be performed for the beams of ${ }^{4} \mathrm{He}$ and ${ }^{6} \mathrm{Li}$ [阿. In conclusion, the systematic measurement of multi-strange hyperons as diagnostic probes of dense nuclear matter at Nuclotron energies has a substantial discovery potential.

\section{BM@N Detector}

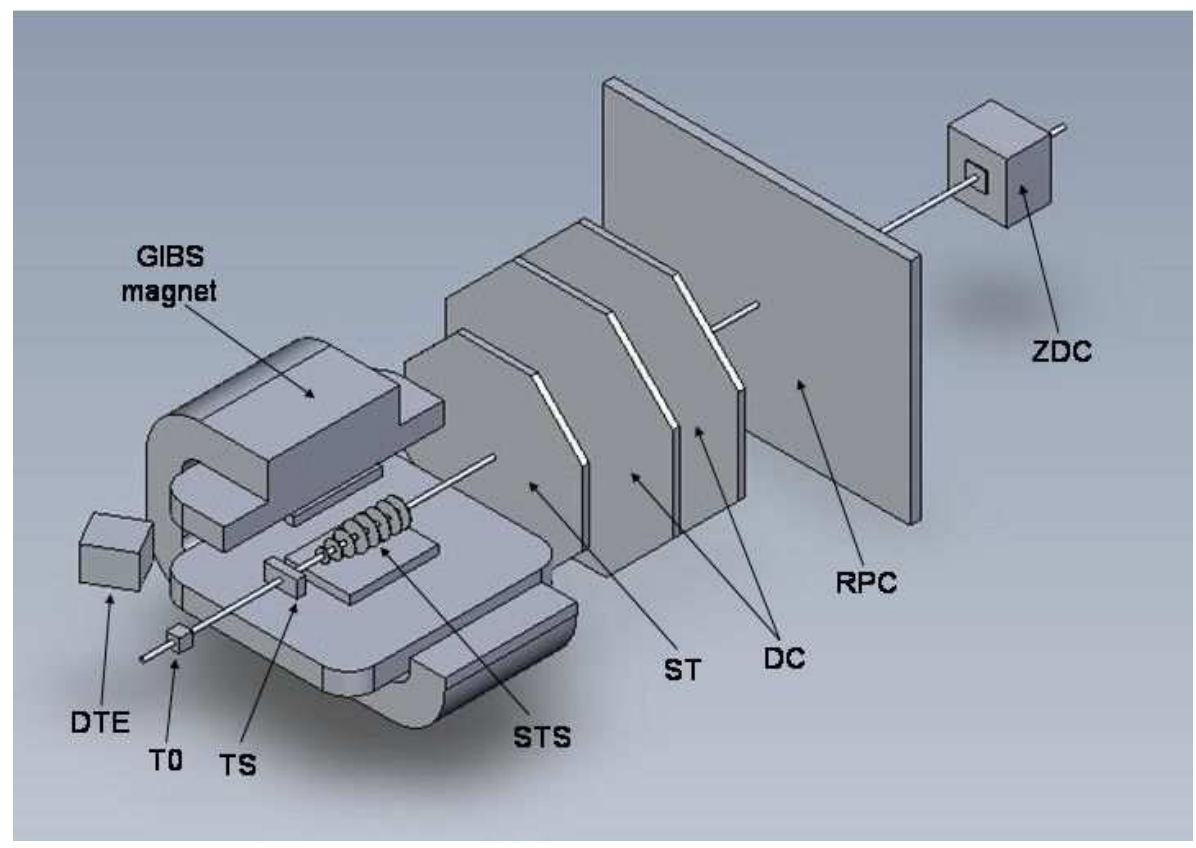

Figure 1: Schematic view of the BM@N setup with the large apperture dipole analyzing magnet SP41. TStarget station, $T 0$ - start diamond detector, $S T S$ - silicon tracker, $S T$ - straw tracker, $D C$ - drift chambers, $R P C$ resistive plate chambers, $Z D C$ - zero degree calorimeter, $D T E$ - detector of transverse energy.

The heart of the BM@N setup is the Silicon Tracking System (STS) (similar to the CBM ST S [6]) placed in the magnetic field with the maximal field integral of $\sim 2 \mathrm{~T} \cdot \mathrm{m}$. The Silicon Tracking 
System comprises 8 stations of increasing size, and will be operated at $-10^{\circ} \mathrm{C}$. Therefore, the STS sits in a thermal enclosure which fits into the gap of the dipole magnet as show in in Fig.1. The double-sided silicon micro-strip sensors will be read out via ultra-thin micro-cables by a freestreaming (i.e. untriggered) front-end-electronics. The sensors, the cables and the electronics are mounted on light-weight carbon ladders which form the stations. At the first stage of the experiment the last 4 stations of the inner tracker can be replaced by single mask foil based GEM technologies or segmented straw tubes. For intermediate tracking two drift chambers from NA48 (delivered from CERN to JINR) can be used [7]. Each drift chamber is composed of eight planes of wires arranged in four views $(x, y, u, v)$ with two planes of staggered wires in each view to resolve the right-left ambiguities. The wire orientations in the $(x, y, u, v)$ views are at multiples of 45 degrees to the horizontal plane and perpendicular to the beam. Sense wire spacing is $1.0 \mathrm{~cm}$; one wire plane extends over $2.40 \mathrm{~m}$ and has 256 sense wires. The coordinate accuracy is found to be $110 \mu \mathrm{m}$ for four planes measurements case. In addition the straw tubes plane developed for muon system of CBM can be used to improve the quality of the tracking. It consists of 3 double-layer planes.
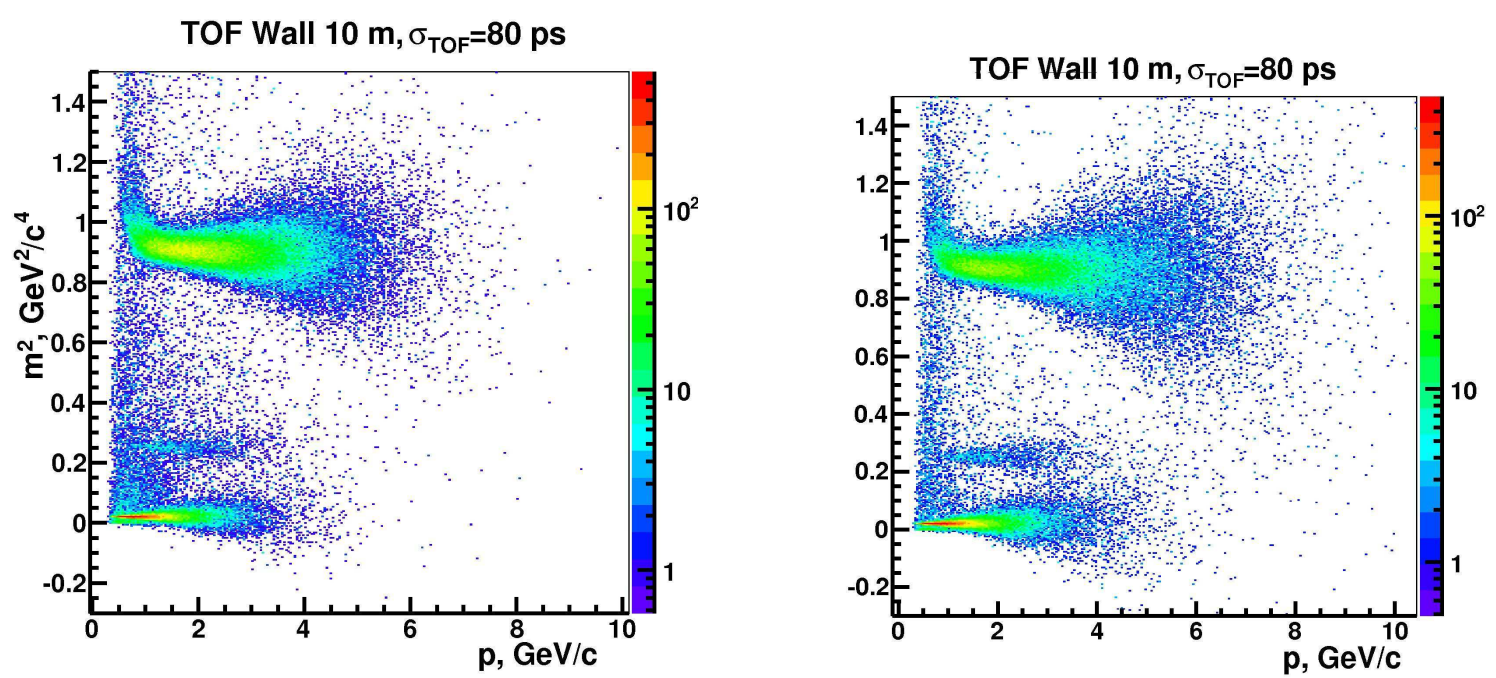

Figure 2: The particle identification using momentum and $T O F$ measurements for central $\mathrm{Au}+\mathrm{Au}$ at $3.5 \mathrm{~A}$ $\mathrm{GeV}$ (left) and $\mathrm{Cu}+\mathrm{Cu}$ at 5.0 A GeV (right).

The experimental setup will be complemented by a TOF- wall based on $m R P C s$ [8, 9, 10] for the particle identification. The simulation performed for the central $\mathrm{Au}+\mathrm{Au}$ at $3.5 \mathrm{~A} \mathrm{GeV}$ and $\mathrm{Cu}+\mathrm{Cu}$ at $5 \mathrm{AGeV}$ collisions shown good particle identification for the timing resolution $80 \mathrm{ps}$ and TOF base $10 \mathrm{~m}$. Figure 2 demonstrates the separation of the secondary particles using $\mathrm{m}^{2}: p$ plot. The precision of the momentum reconstruction in silicon tracker is $\Delta p / p=2 \%$. The reduce of the TOF base will decrease of the quality of the particle identification. However, still $8 \mathrm{~m}$ of the base is enough to select the particles at the energies of Nuclotron. The working conditions of proposed experiment for the $T 0$ detector require the use of diamond pixel or strip detector because of high radiation hardness. Two technologies are considered, namely, mono-crystalline pad diamond detector applied recently for HADES [11] and poly-cristalline double-sided strip module developed for ATLAS [12].

The centrality of the event will be measured using zero degree calorimeter $(Z D C)$. For this 
purpose the reassembled the WA98 ZDC [13] will be used. This hadron calorimeter consists of lead/scintillator sandwich modules with the sampling satisfying the compensating condition. The cross section of the module is $15 \times 15 \mathrm{~cm}^{2}$. The energy resolution is about $50 \% / \sqrt{E(\mathrm{GeV})}$ for hadrons. The transverse cross section of $Z D C$ will be $150 \times 150 \mathrm{~cm}^{2}$. The independent estimation of the centrality can be performed using DTE based on PINOT electromagnetic calorimeter [14]. Also the multiplicity in TOF detector can be used as an independent way to measure the centrality.

The $D A Q$ system should be able to store and analyze the data obtained from the different detectors both in free-streaming (STS) and triggered (for instance, RPCS or ZDC) modes. The high counting rate expected during the experiment requires the development of high-speed methods, algorithms and software tools of parallel processing for solving problems on multiprocessor and distributed computing complexes, including the use of Grid-technologies. The use of SIMD instructions, simultaneous multi-threading (SMT), algorithmic languages Ct, OpenCL and CUDA for solving problems on the multi-core, multiprocessor distributed computer complexes. These methods, algorithms and software will be used for the charged particles momenta reconstruction with the high accuracy and their identification, for the simulation of the physical processes in heavy ion collisions at Nuclotron energies, the calibration and alignment of the setup detectors. The data taking and analysis will be performed using distributed systems for the data processing.

\section{Simulation status}

Experimentally the $\Lambda, \Xi$, and $\Omega$ hyperons can be identified via the topology of their weak decays $\left(\Lambda \rightarrow p \pi^{-}, \Xi \rightarrow \Lambda \pi, \Omega \rightarrow \Lambda K\right)$. The simulation have been performed using the UrQMD event generator, the BM@N detector model in the GEANT transport code, and the event reconstruction algorithms in the CBMRoot software package. The STS detector comprises 8 stations of double-sided silicon micro-strip detectors (pitch $60 \mu \mathrm{m}$, stereo angle $7.5^{\circ}$ ) located in the field of a large aperture dipole magnet SP41.


Figure 3: Reconstructed $K_{s}^{0}$ mesons (left) and $\Lambda$ hyperons (right) for central Au+Au collisions at $4 \mathrm{~A} \cdot \mathrm{GeV}$ using the UrQMD event generator.

The results of the simulations for the configurations of STS similar to the CBM case (8 stations, last 4 suited to the gap of the SP41 magnet) are shown in Fig. 3 which depicts the invariant mass spectra for $K_{s}^{0}$ mesons and $\Lambda$ hyperons for central $\mathrm{Au}+\mathrm{Au}$ collisions at $4 \mathrm{~A} \cdot \mathrm{GeV}$. The anal- 
ysis is based on topological cuts only, without particle identification via time-of-flight determination. The combinatorial background can be further reduced by identifying the decay protons by a time-of-flight measurement. One can see the large efficiencies of the reconstruction and good signal/background ratios.
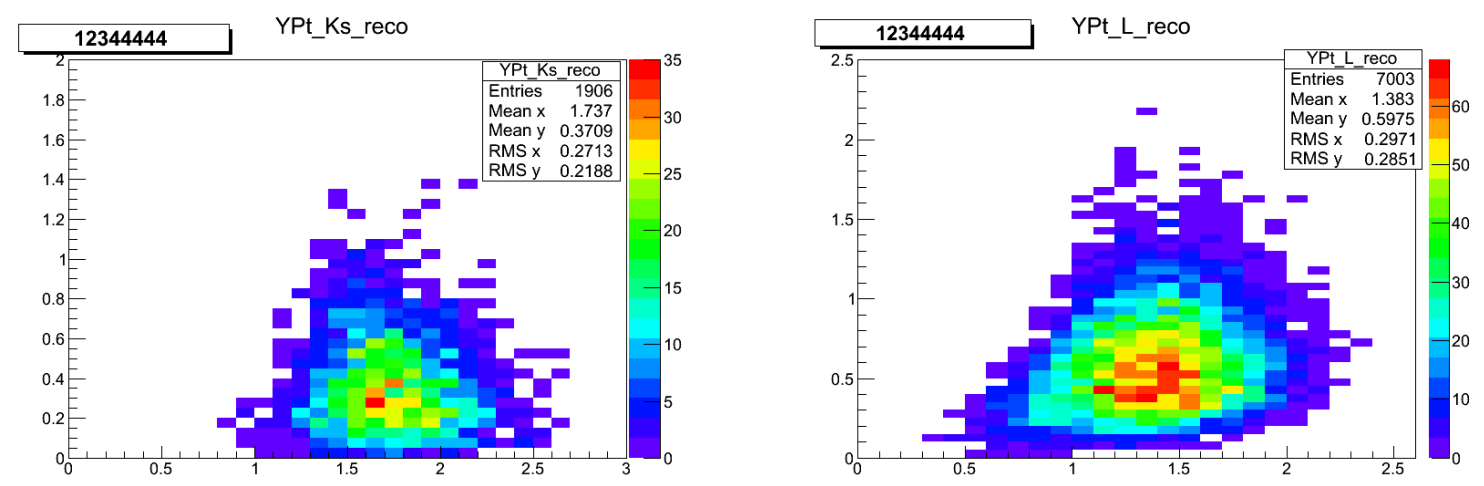

Figure 4: The $y-p_{T}$ acceptances for $K_{s}^{0}$ mesons (left) and $\Lambda$ hyperons (right) for central Au+Au collisions at $4 \mathrm{~A} \cdot \mathrm{GeV}$.

The $y-p_{T}$ acceptances for $K_{s}^{0}$ mesons (left) and $\Lambda$ hyperons (right) for central $\mathrm{Au}+\mathrm{Au}$ collisions at $4 \mathrm{~A} \cdot \mathrm{GeV}$ are shown in Fig. $\bigoplus$. One can see that the acceptances are large enough.


Figure 5: The $y-p_{T}$ distributions for $K^{+}$mesons from the central $\mathrm{Au}+\mathrm{Au}$ collisions at $4 \mathrm{~A} \cdot \mathrm{GeV}$ from left to right: UrQMD events within BM@N setup acceptance, reconstructed events using STS and TOF, the reconstruction efficiency.

The $y-p_{T}$ distributions for $K^{+}, \pi^{-}$mesons and protons from the central $\mathrm{Au}+\mathrm{Au}$ collisions at 4 A.GeV for UrQMD events within BM@N setup acceptance, reconstructed events using STS and $T O F$, the reconstruction efficiency are shown in Figs 5 , 6 and 7 in the left, central and right panels, respectively. One can see that the acceptances are quite large and the reconstruction efficiencies at low $p_{T}$ are large enough.

The simulation framework is still under development. The optimization of the BM@N detector is continued.

\section{Status of the detector}

The measurements with $3.42 \mathrm{~A} \cdot \mathrm{GeV}$ carbon and 1.0-4.0 A.GeV deuteron beams in 2011- 

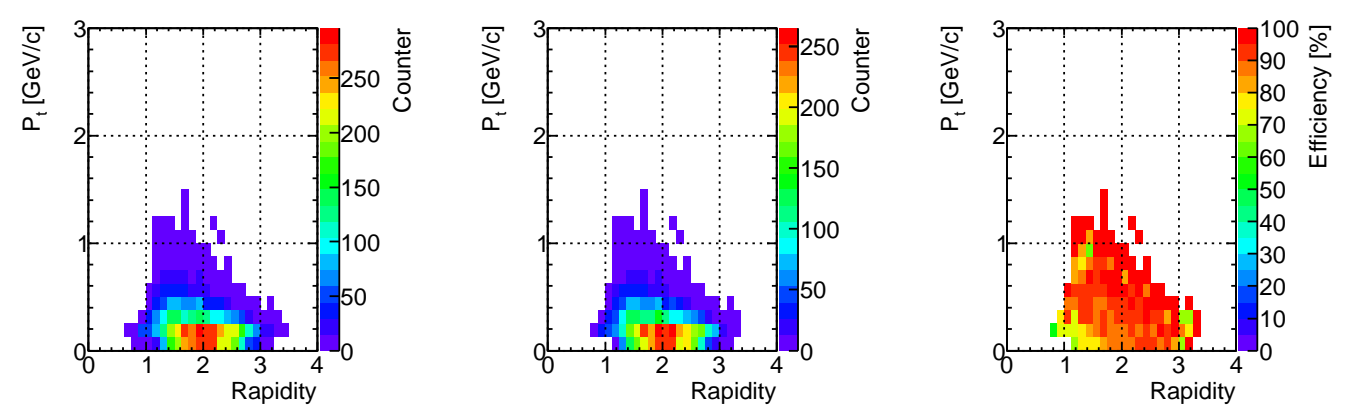

Figure 6: The $y-p_{T}$ distributions for $\pi^{-}$mesons from the central $\mathrm{Au}+\mathrm{Au}$ collisions at $4 \mathrm{~A} \cdot \mathrm{GeV}$ from left to right: UrQMD events within BM@N setup acceptance, reconstructed events using STS and TOF, the reconstruction efficiency.
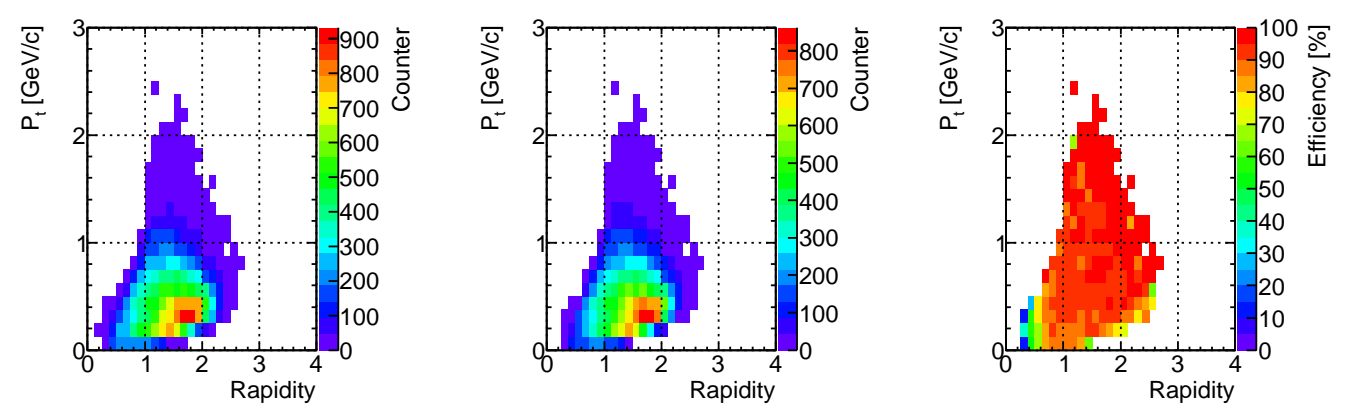

Figure 7: The $y-p_{T}$ distributions for protons from the central $\mathrm{Au}+\mathrm{Au}$ collisions at $4 \mathrm{~A} \cdot \mathrm{GeV}$ from left to right: UrQMD events within BM@N setup acceptance, reconstructed events using STS and TOF, the reconstruction efficiency.

2012 demonstrated the feasibility of the experiment with the existing beamlines. On the base of these measurements the technical requirements to the Nuclotron parameters, beam transportation conditions and experimental cave details were formulated.

The experimental zone is now under reconstruction. New beam stopper, target station and beam pipe is in preparation. We are planning to enlarge the aperture of the BM@N magnet up to $1 \mathrm{~m}$ in the vertical direction. The calculations of the magnetic field for new magnet design are progress now.

The simulation shown that the counting rate at $m R P C s$ requires the use of warm float glass techniques use. Such detectors have been developed at IHEP and will be tested in December at U70 Accelerator complex using specially constructed muon beam line.

The scintillation fiber hodoscopes based on the use multi-anode PMTs H6568 are under construction and testing.

The WA98 ZDC calorimeter will be delivered from CERN to JINR soon. On its base the ZDC for BM@N experiment will be constructed.

The work on the electronics and $D A Q$ concept is in progress. 


\section{Conclusion}

The major scientific mission for the BM@N spectrometer with inner tracker is the the measurements of the (sub)threshold cascade hyperons production in order to obtain the information on the nuclear matter equation of state.

However, even with the start version of the BM@N setup (without inner tracker) very interesting scientific program can be realized. This program will include the studies of

- in-medium effects for strangeness and vector mesons decaying in hadron modes;

- flows, polarizations, vorticity and azhimuthal correlations of hadrons;

- femtoscopy for different hadrons (and photons);

- $N N$ - and $N A$ - interactions as the reference for $A A$ - collisions.

BM@N experiment has very high discovery potential which can provide new insight of the problem. This experiment can be the frontier for the large scale experiments like CBM, MPD, PANDA and SPD inventing new detector technologies for JINR home experiments.

\section{References}

[1] P.Senger and V.Friese, CBM Report 2011-1; https://www.gsi.de/documents/DOC-2011-Aug-29-1.pdf.

[2] B. Friman, W. Nörenberg and V.D. Toneev, Eur. Phys. J. A3 (1998) 165.

[3] A. Andronic et al., Nucl.Phys. A837 (2010) 65, arXiv:0911.4806 [hep-ph].

[4] A. Andronic, P. Braun-Münzinger, J. Stachel and H. Stöcker, Phys. Lett. B695 (2011) 203, arXiv:1010.2995v1 [nucl-th].

[5] Yu.A.Batusov, J.Lukstins, L.Majling and A.N.Parfenov, Phys.Elem.Part.Atom.Nucl. 36 (2005) 169.

[6] J.M.Heuser, Nucl.Instrum. and Meth. A582 (2007) 910.

[7] D.Bederede et al., Nucl.Instrum. and Meth. A367 (1995) 88; I.Augustin et al., Nucl.Instrum. and Meth.A403 (1998) 472.

[8] I.Deppner et al., Nucl.Instrum. and Meth.A661 (2012) S121.

[9] V.V. Ammosov et al., Instrum.Exp.Tech.53 (2010) 175.

[10] Y.Wang et al., Nucl.Instrum.Meth. A661 (2012) S134.

[11] J.Pietraszko et al., Nucl.Instrum.Meth. A618 (2010) 121.

[12] M.Mikuz et al., Nucl.Instrum.Meth. A579 (2007) 788, V.Adam et al., Eur.Phys.J. C33 (2004) s1014, Doi:10.1140/epjcd/s2004-03-1798-6.

[13] V.A.Arefiev et al., JINR Rapid Communications 5[79]-96 (1996) 15.

[14] E.Chiavassa et al., IEEE Trans. Nucl.Sci.33 (1986) 209. 